\title{
Converting a Bulk Radiation-Hardened BiCMOS Technology into a Dielectrically-Isolated Process ${ }^{*}$
}

\author{
M. DeLaus ${ }^{\dagger}$, D. Emily ${ }^{+}$, B. Mappes ${ }^{+}$, R. Pease ${ }^{\ddagger}$ \\ †Analog Devices Semiconductor \\ Wilmington, MA \\ ${ }^{+}$Naval Surface Warfare Center \\ Crane, IN \\ $~^{*}$ RLP Research, Inc. \\ Albuquerque, NM
}

\begin{abstract}
Development of a dielectrically-isolated (DI) radiation-hardened BiCMOS process is reported. The process is fabricated on a bonded-wafer silicon-on-insulator (SOI) substrate and employs deep trenches for lateral device isolation.

\section{INTRODUCTION}

RBCMOS (Radiation-hardened Bipolar CMOS) is a 10-volt, junction-isolated, radiation-hardened $\mathrm{BiCMOS}$ process developed at Analog Devices. It is a radiation-hardened version of Analog Devices' commercial Advanced Bipolar CMOS (ABCMOS) process. Both of these processes are designed for advanced mixed-signal circuits such as high-performance D/A and $A / D$ converters. To improve the dose-rate performance of the process, the DI version is being developed. The isolation scheme employed is similar to that used on state-of-the-art commercial complementary-bipolar processes ${ }^{1,2}$. The starting material is a bonded wafer SOI substrate. Full oxide isolation is achieved with deep trenches that are filled with a combination of oxide and polysilicon. Excellent transistor yield has been demonstrated. The DI process exhibits improved performance in high dose-rate environments, including higher upset thresholds, and improved recovery times. The addition of the oxide isolation did not introduce any radiation sensitivities.
\end{abstract}

\section{PROCESS DESCRIPTION}

A device cross section of RBCMOS-DI appears in Figure 1. The starting material is a commercially available bonded-wafer SOI substrate formed by the bond, lap-back, and polish technique ${ }^{3}$. The starting SOI layer is $2.0 \mu \mathrm{m}$ thick with a $1.0 \mu \mathrm{m}$ buried oxide. The bonded-wafer material was selected over

* Work supported by the United States Army Space and Strategic Defense Command and the Defense Nuclear Agency. other types of SOI due to its superior crystallographic quality and thicker SOI and buried-oxide layers. A minimum silicon layer thickness of approximately $1.5 \mu \mathrm{m}$ is required to process the $\mathrm{N}+$ buried layer prior to epi deposition and a $1.0 \mu \mathrm{m}$ buried oxide substantially reduces the parasitic substrate capacitances. The bonded-wafer was simply substituted for the conventional bulk substrate. No special processing was required to accommodate the SOI substrate.

\section{A. PROCESS FLOW}

After the $\mathrm{N}+$ buried layer is implanted and diffused into the SOI layer, a P-type epitaxial layer is grown. Prior to trench processing, the deep $\mathrm{N}+$ collector plugs and $\mathrm{N}$-wells are implanted and diffused. The trenches are $2.0 \mu \mathrm{m}$ wide, $5.5 \mu \mathrm{m}$ deep, and bottom on the buried oxide to yield full dielectric isolation. The trench is lined with oxide and filled with polysilicon. The trench etch and refill process was optimized to minimize crystal defect formation at both the top and bottom corners of the trench.

Following planarization, a thick thermal field oxide is grown. After the P-base implant, the $\mathrm{N}+$ polysilicon emitter is formed. The CMOS portion of the process consists of a $350 \AA$ gate oxide and self-aligned polysilicon gate electrode. Borophosphosilicate glass (BPSG) is then deposited and thin-film resistors are formed. Double level metal and passivation complete the processing.

Table 1 provides a summary of the electrical performance of RBCMOS-DI. The performance of the junction isolated (J) and DI versions of RBCMOS are the same.

\section{B. RBCMOS-DI TEST CHIP}

Process experiments were conducted using the RBCMOS-DI 


\section{NPN PMOS NMOS}

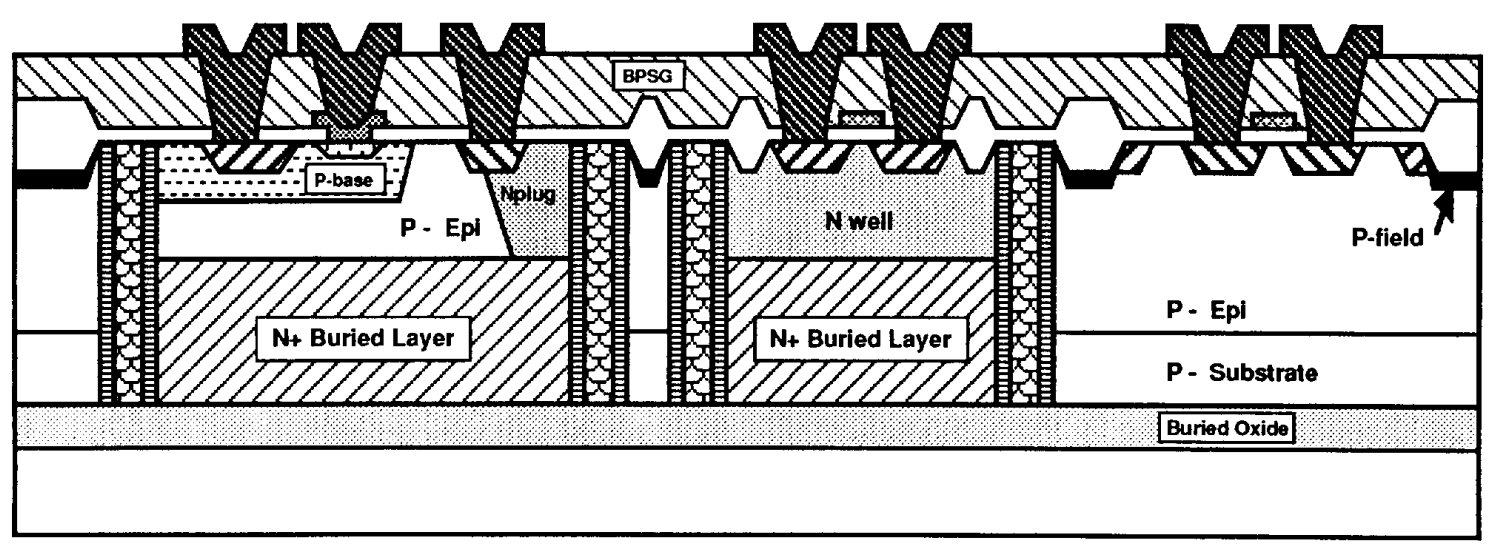

FIGURE 1. Device cross-sections of RBCMOS-DI NPN, NMOS, and PMOS transistors

\begin{tabular}{|c|c|c|}
\hline Bipolar & NPN & PNP \\
\hline HFE & 200 & 100 \\
\hline BVceo (V) & $>13$ & $>15$ \\
\hline $\mathrm{VA}(\mathrm{V})$ & 40 & 25 \\
\hline$\Delta$ Vbe $(\mathrm{mV})$ & 0.5 & $-\cdots$ \\
\hline $\mathrm{Ft}(\mathrm{GHz})$ & 3.0 & 0.1 \\
\hline 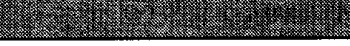 & 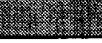 & 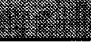 \\
\hline MOS & NMOS & PMOS \\
\hline $\mathrm{Vt}(\mathrm{V})$ & 0.8 & -0.8 \\
\hline BVdss (V) $\mathrm{L}=2 \mu \mathrm{m}$ & $>8.0$ & $>10.0$ \\
\hline $\mathrm{L}=5 \mu \mathrm{m}$ & 15 & 13 \\
\hline Gate Delay (psec) & 450 & 450 \\
\hline 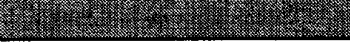 & \multicolumn{2}{|c|}{ 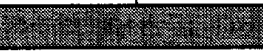 } \\
\hline \multicolumn{3}{|l|}{ ThinFilm } \\
\hline Sheet Resistance & \multicolumn{2}{|c|}{$1,000 \mathrm{ohms} /[]$} \\
\hline Temperature Coefficient & \multicolumn{2}{|c|}{$-50 \mathrm{ppm} /{ }^{\circ} \mathrm{C}$} \\
\hline \multicolumn{3}{|l|}{ Zener Diode } \\
\hline BVzen & \multicolumn{2}{|c|}{$4.6 \mathrm{~V}$} \\
\hline
\end{tabular}

TABLE 1. RBCMOS-DI Device Parameters

test chip. The mask set contains a number of test structures and devices designed to evaluate the yield, electrical performance, and radiation response of the process. Small circuits were also included on the mask set. Chains of NPN transistors were used to monitor the defect density. Chains are a series of transistors connected in parallel. Six different chain sizes were used in this study, the largest one containing $2001 \mathrm{X}$ NPN transistors (emitter area $2 \mu \mathrm{m} \times 15 \mu \mathrm{m})$. The total emitter area of the $200 \mathrm{X}$ chain is $6,000 \mu \mathrm{m}^{2}$.

Both junction-isolated and trench-isolated chains were included on the mask set. It is important to separate the defects originating in the SOI material from those caused by the interaction of the trench and buried-oxide layer. The JI chains are useful for evaluating the quality of the bonded-wafer substrates. Over $70 \%$ of the mask set was devoted to defect density monitoring structures. This allowed a great amount of data to be collected, which is important for statistically valid results. As defect levels are reduced, more area must be sampled to obtain an accurate yield figure.

In order to investigate the influence of the NPN layout on the device yield, 6 different trenched NPN designs were implemented on the test chip. Since the transistors are affected by defects located in the area of the base below the emitter, the proximity of the trench isolation to this region is important. Some of the layouts had the trenches conservatively spaced further away from the emitter, while others had the emitter as close as $3 \mu \mathrm{m}$ from the trenches.

The trenched NPN designs were designated as follows: TR has the trench outside the base and collector; TR-NPLG has a ring of N-plug between the trench and the base area; TR-Thox has thick-oxide grown over the trench region; TR-walled has the trench walling the base on 3 sides; TR-nonwalled has the base spaced $2 \mu \mathrm{m}$ from the trench; TR-walled/sp has the base walling the trench on 3 sides but with the emitter pulled in $10 \mu \mathrm{m}$ from the edge of the base. 


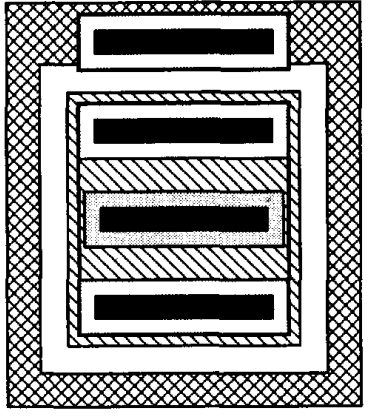

$\mathrm{JI}$

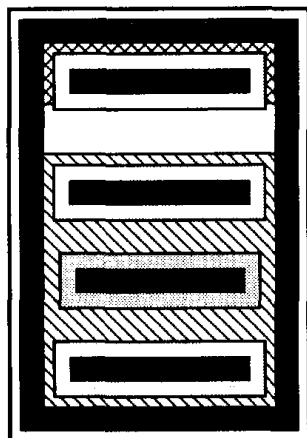

Trench/walled

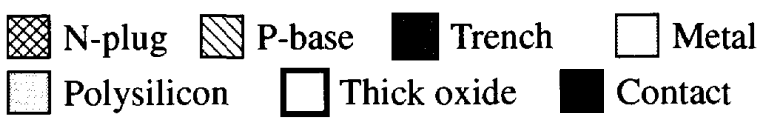

FIGURE 2. Transistor layouts of the JI and TR-walled NPN devices.
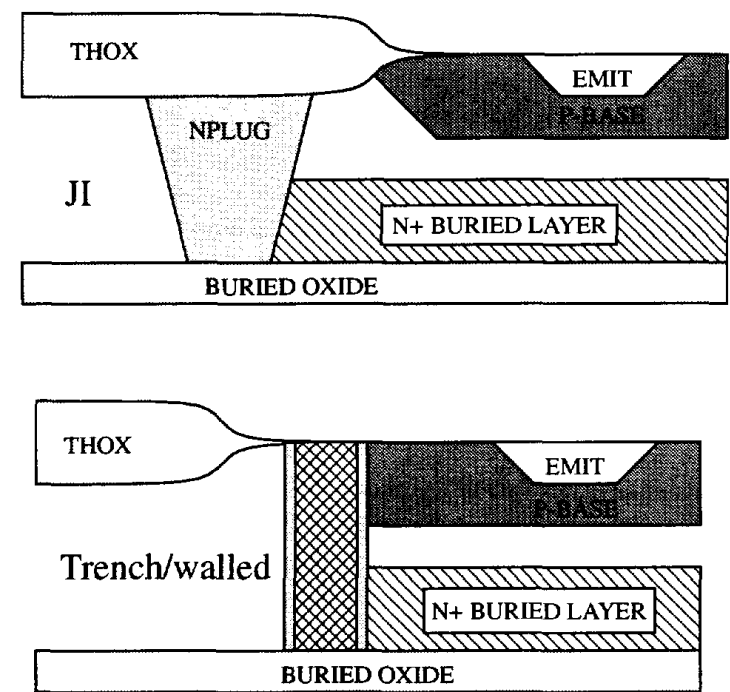

FIGURE 3. Transistor cross-sections of the JI and TRwalled NPN devices

The layout of the JI NPN and TR-walled NPN is shown in Figure 2. The TR-walled device was the most aggressive layout. As can be seen in the figure, the P-base is walled on 3 sides by the trench. Figure 3 contains partial cross-sections of these two devices.

\section{RESULTS and DISCUSSION}

The major challenge in developing a DI process employing trench isolation and a SOI substrate is to minimize the crystallographic defects that can be formed at the corners of the trench. These defects can result from process induced stress caused by thermal oxidations. The stress results because thermal oxides have a greater volume than the silicon they consume during their formation. The defects can serve as sites for the formation of collector-emitter pipes, which cause excessive collector-emitter leakage in bipolar transistors.

To assess the level of the process-induced defects, measurements were made of the transistor chains. To sample the most area, all 125 sites were probed on each wafer processed. During the chain probing, various NPN device parameters were measured, including $\mathrm{HFE}, \mathrm{BV}_{\mathrm{CEO}}, \mathrm{BV}_{\mathrm{CBO}}$, and $\mathrm{I}_{\mathrm{CEO}}$. Transistor $\mathrm{BV}_{\mathrm{CEO}}$ was chosen as the criteria for determining if a chain was good or bad. Transistor $B V_{C E O}$ is known to be very sensitive to the presence of defects ${ }^{4}$. If a defect propagates up from the collector of a device through the emitter, it can provide a leakage path that will decrease the apparent breakdown of the device. Only a single device in a transistor chain needs to be bad for the chain to fail. A good chain had a $B_{C E O}$ of $\geq 10$ volts.

\section{A. ELECTRICAL TEST RESULTS}

Several wafer lots have been processed with the RBCMOS-DI test mask. Each lot contained from 10 to 14 wafers. Process experiments examined different trench process schemes, primarily focusing on the trench sidewall oxide. For the process experiments, the control split was the JI chains fabricated on the standard bulk substrate. A minimum of 2 standard bulk wafers were included in each lot.

After the lot processing was completed, the transistor chains on each wafer were mapped. There are 125 sites per wafer. A chain was considered defective if the $\mathrm{BV}_{\text {ceo }}$ fell below 10 volts. When all of the wafer mapping had been completed, the overall chain yield for each of the process splits was calculated.

A simple Poisson model was used to determine the defect density based on the electrical yield and area of the chains. The yield model is:

$$
\mathbf{Y}=\mathbf{e}^{-D_{0}} \mathbf{A}
$$

Where $Y$ is the chain yield, $D_{0}$ is the defect density in $\mathrm{cm}^{-2}$, and $\mathrm{A}$ is the chain area in $\mathrm{cm}^{2}$. Solving for the defect density results in:

$$
D_{0}=-\ln Y / A
$$

Given the emitter area of the 200 transistor chain $\left(6,000 \mu \mathrm{m}^{2}\right)$, and assuming a yield calculation based on 250 total possible sites ( 2 wafers per split), the minimum detectable defect density is 67 defects $/ \mathrm{cm}^{2}$. It should be noted that a $100 \%$ chain 


\begin{tabular}{|l|c|c|c|}
\hline \multicolumn{1}{|c|}{ Layout } & $\begin{array}{c}\text { Substrate } \\
\text { Type }\end{array}$ & $\begin{array}{c}\text { \# bad sites } \\
(250 \text { total })\end{array}$ & $\begin{array}{c}\text { Defect } \\
\text { density } \\
\left(\mathrm{cm}^{-2}\right)\end{array}$ \\
\hline II & Bulk & 1 & 67 \\
\hline JI & Bonded & 1 & 67 \\
\hline TR-THOX & Bonded & 27 & 1905 \\
\hline TR-NPLG & Bonded & 5 & 337 \\
\hline TR & Bonded & 0 & $<67$ \\
\hline TR-Nonwalled & Bonded & 1 & 67 \\
\hline TR-Walled & Bonded & 3 & 201 \\
\hline TR-Walled/sp & Bonded & 3 & 201 \\
\hline
\end{tabular}

TABLE 2. Defect Density results for the process experiments as a function of transistor layout and substrate.

yield does not mean that there are no defects, only that the defect level is below the resolution of the test structures. It is assumed in the defect density calculations that all of the yield loss was due to trench/SOI induced defects. Other problems such as electrical misprobes or metal shorts could also cause the transistor chains to appear to be defective, but the levels of these problems should be well below the trench/SOI defect levels except perhaps in the instances where only one or two defective chains are observed.

The optimum sidewall oxide splits had defect densities on the DI chains comparable to the JI chains fabricated on the bulk wafers. The defect density results are summarized in Table 2 . One important observation is that the JI transistor chains had the same yield/defect densities whether fabricated on a bulk or a bonded-wafer substrate. This suggests that the material quality of the bonded-wafer SOI substrate is equal to that of bulk silicon.

The only trenched NPN layout that produced poor results was the case where the thick field oxide is grown directly over the trench. This layout resulted in defects being generated at the top corners of the trenches, adversely affecting the chain yield. In all of the other layouts, the thick field oxide was pulled back $1 \mu \mathrm{m}$ from the trench.

Emitter defect densities of $<400 \mathrm{~cm}^{-2}$ would make possible the manufacture of even large area circuits. Circuits with die sizes greater than $75,000 \mathrm{mils}^{2}$, containing over $1,000 \mathrm{NPN}$ transistors would have device yields in excess of $95 \%$.

\section{B. RADIATION TEST RESULTS}

The greatest risk in developing RBCMOS-DI was that the introduction of the insulating oxides would also create a sensitivity to ionizing radiation. To minimize this risk, the transistor layouts were conservative. Critical emitter-base junctions were

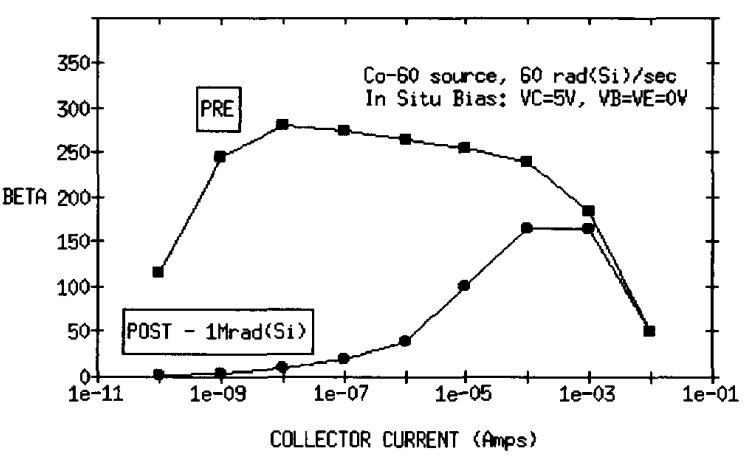

FIGURE 4. Total-dose response of RBCMOS JI $2 \times 15$ NPN transistor

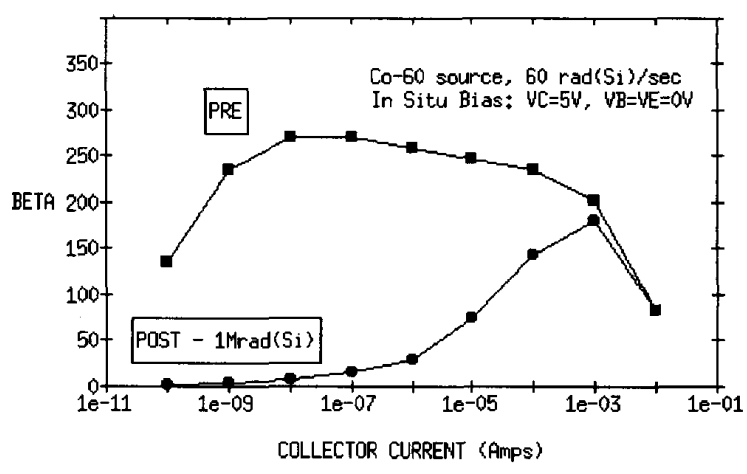

FIGURE 5. Total-dose response of RBCMOS-DI TRwalled 2x15 NPN transistor

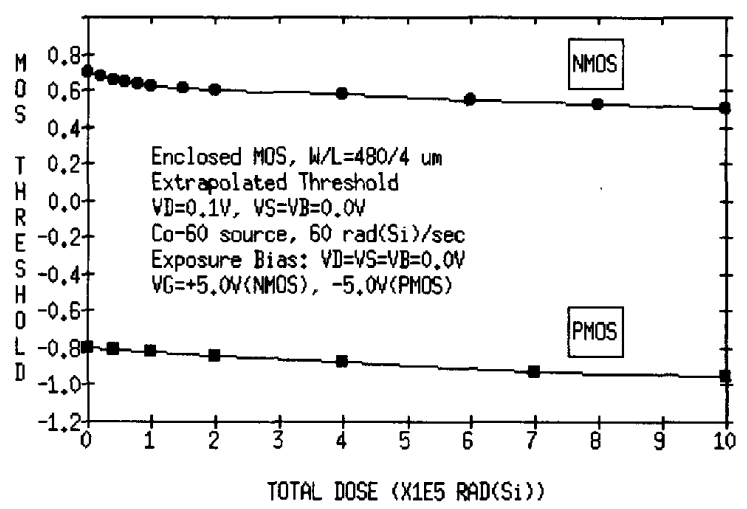

FIGURE 6. Total-dose response of RBCMOS-DI MOS transistors 


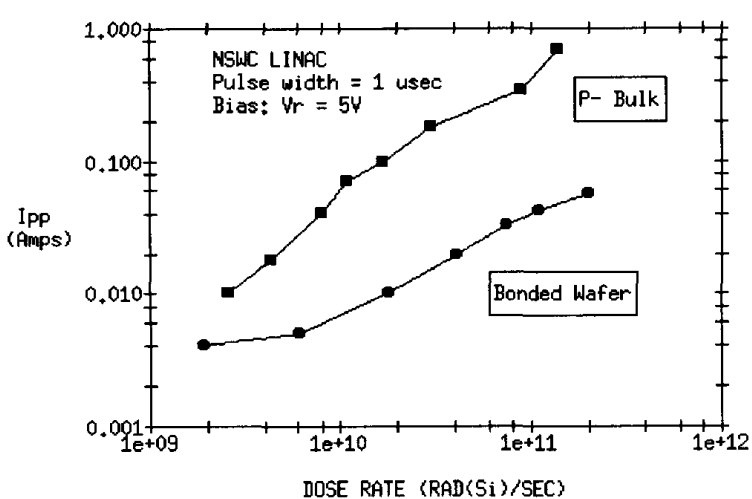

FIGURE 7. Photocurrent diode response for device built on bulk and bonded-wafer substrates.

not walled by the trench. To verify that the total-dose performance of the process was not compromised, both JI and DI NPN transistors were irradiated. Figures 4 and 5 illustrate the total-dose response of the JI and DI devices, respectively. The response is essentially the same for both device types, and is typical to that seen on advanced polysilicon-emitter devices. The pre- and post-radiation collector-emitter leakage current was also monitored during the testing. No significant increase in the leakage current was noted. For completeness, the totaldose response of the RBCMOS-DI MOS devices is shown in Figure 6. Back-channel leakage is not an issue since the source/drain diffusions are much shallower $(<0.4 \mu \mathrm{m})$ than the total silicon film thickness.

The obvious benefit of DI is improved performance in high dose-rate environments. DI eliminates the NPN collector/substrate and the PMOS well/substrate junctions, the major source of photocurrents. To quantify this improvement, photocurrent diodes fabricated on bulk and bonded wafers were tested at the NSWC-Crane LINAC. The diodes were formed with the Nsource/drain diffusion and the P-type epi/substrate. The diode area was $1.6 \times 10^{5} \mu \mathrm{m}^{2}$. The results are shown in Figure 7. At higher dose-rates, the photocurrent is reduced by an order of magnitude on the diodes built on a bonded wafer.

RBCMOS-DI and JI versions of a comparator and D/A converter switching cells have been fabricated and are being tested to evaluate the circuit-level performance of the process. Benefits of an insulating substrate have been previously reported ${ }^{5}$. The reduced photocurrent collection results in increased circuit upset levels and improved recovery times. While photocurrent tails linger for a few microseconds on the bulk diodes, photocurrents subsided in only a few tenths of microseconds on the bonded wafer diodes.

Latchup is nonexistent on DI processes, as each transistor is isolated in an oxide tube. Single-event effects are expected to be minimized on RBCMOS-DI because the charge collection volumes are greatly reduced.

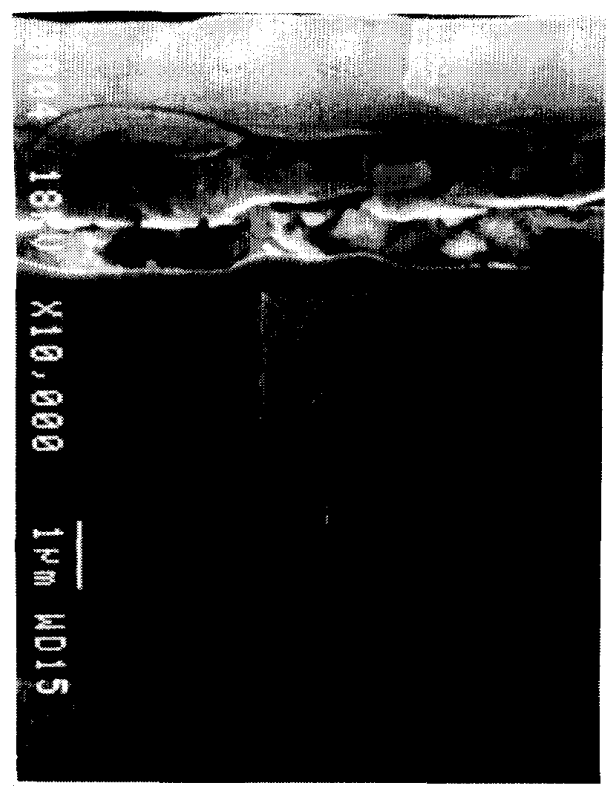

FIGURE 8. SEM cross-section of RBCMOS-DI trench/ buried oxide region

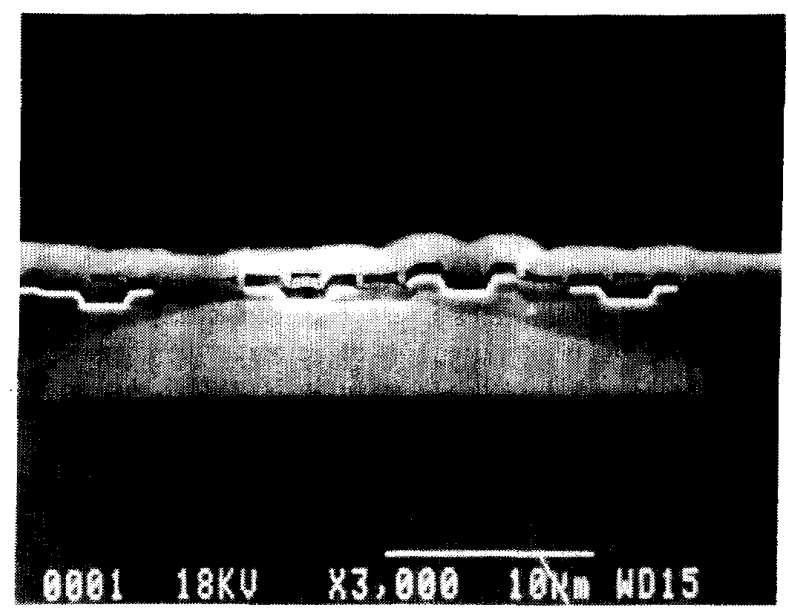

FIGURE 9. SEM cross-section of RBCMOS-DI trench/ walled NPN transistor

\section{Physical Analysis}

In addition to the electrical and radiation evaluation of the RBCMOS-DI structures, a physical analysis was also conducted. This primarily consisted of examining device crosssections with a SEM. Some of these results are shown in Figures 8 and 9. Figure 8 is a cross-section of a trench showing the oxide sidewall and the polysilicon refill. The buried oxide is 
also visible. The metal running over the top of the trench demonstrates the excellent trench refill planarization and step coverage. Figure 9 is a full NPN cross-section.

\section{CONCLUSIONS}

A radiation-hardened dielectrically-isolated BiCMOS process has been developed by retrofitting DI to an existing radiationhardened JI process. The isolation technique employed is similar to that used on advanced commercial complementary-bipolar processes. Trench/substrate induced defects are sensitive to the device layout and process flow. Optimization of the trench and post-trench processing and the device layouts has reduced the defect densities to acceptable levels. The defect density levels obtained are consistent with the economic manufacture of VLSI circuits. The dose-rate performance of the process has been improved without compromising the total-dose hardness.

\section{ACKNOWLEDGMENTS}

The authors wish to thank Dale Platteter/NSWC Crane and Lew Cohn/DNA for their continued support of this work. This paper is dedicated to Ed Enlow who made substantial contributions to this project.

\section{REFERENCES}

1. S. Feindt, J-J. J. Hajjar, J. Lapham, and D. Buss, "XFCB: A High Speed Complementary Bipolar Process on Bonded SOI", BCTM Proceedings, pp. 264-267, 1992

2. C. Davis, G.Bajor, J.Butler, T. Crandell, J.Delgado, T.Jung, Y. Khajeh-Noori, B. Lomenick, V. Milam, H. Nicolay, S. Richmond, T. Rivoli, "UHF-1: A High Speed Complementary

Bipolar Analog Process on SOI", BCTM Proceedings, pp. 260$263,1992$.

3. T. Abe, M. Nakano and T. Itoh, "Silicon Wafer-Bonding Process Technology for SOI Structures", Proceedings of the 4th International Symposium on Silicon-on-Insulator Technology and Devices, D. N. Schmidt ed., May 1990, Montreal, The Electrochemical Society, NJ.

4. K. Ravi, "Imperfections and Impurities in Semiconductor Silicon”, John Wiley\& Sons, Inc., New York, New York, pp. 257-258, 1981.

5. D. Platteter, and T. Cheek, "The Use of Multiple Oxygen Implants for Fabrication of Bipolar Silicon-on-Insulator Integrated Circuits", IEEE Trans. Nuc. Sci., Vol. 35 No. 6, pp.

1350-1354, 1988. 